\title{
A aplicação de 1-metilciclopropeno (1-MCP) aumenta a conservação pós-colheita de peras
}

\author{
1-methylcyclopropene (1-MCP) application increases post-harvest storage of pears
}

\author{
Mateus da Silveira Pasa ${ }^{*}$, Horacy Fagundes da Rosa Júnior ${ }^{2}$, Émerson de Franceschi ${ }^{2}$, Robson \\ Rodrigues Pereira², Juan Saavedra del Aguila ${ }^{3}$, José Carlos Fachinello² \& Carina Pereira da Silva ${ }^{2}$ \\ ${ }^{1}$ Oregon State University/Mid-Columbia Agricultural Research and Extension Center, Hood River, OR. *Autor para correspondência: \\ mateus.pasa@oregonstate.edu. \\ ${ }^{2}$ Universidade Federal de Pelotas, Pelotas, RS, Brasil. \\ ${ }^{3}$ Universidade Federal do Pampa, Bagé, RS, Brasil.
}

Submissão: 09/03/2017 / Aceite: 09/05/2018

\begin{abstract}
RESUMO
Para manutenção da qualidade e aumento no tempo de armazenamento de frutas climatéricas como a pera, uma das estratégias é a utilização do 1-metilciclopropeno (1-MCP), que reduz os efeitos do etileno, o qual é responsável pelo amadurecimento dos frutos. Objetivou-se a avaliação da influência do 1-MCP na qualidade pós-colheita de peras 'Abate Fetel', 'Packham's' e 'Tenra' armazenadas em câmara fria. As frutas foram tratadas com 1-MCP no interior de caixas plásticas vedadas, à temperatura de $20 \stackrel{\circ}{\circ}$, por 14 horas, sendo em seguida transferidas para câmara fria $\left(0{ }^{\circ} \mathrm{C} \pm 1{ }^{\circ} \mathrm{C}\right.$ e $85 \% \pm 5 \%$ UR), onde permaneceram por períodos de 30,60, 90 e 120 dias. As variáveis analisadas foram firmeza de polpa, índice DA, pH do suco, acidez titulável e sólidos solúveis. Observou-se que o 1-MCP é eficiente na manutenção da firmeza de polpa dos frutos das cultivares estudas, armazenadas em câmara fria. Além disso, a degradação de clorofila de peras 'Abate Fetel' e 'Packham's', armazenadas em câmara fria, foi reduzida com a utilização do 1-MCP.
\end{abstract}

PALAVRAS-CHAVE: Pyrus communis, etileno, maturação, qualidade de fruto.

\begin{abstract}
One of the strategies to maintain fruit quality and increase storage time of climacteric fruit such as pear is the use of 1-methylcyclopropene (1-MCP), reducing the effects of ethylene, which is the main responsible for fruit ripening. The aim of this study was to evaluate the influence of 1-MCP on post-harvest fruit quality of 'Abate Fetel', 'Packham's' and 'Tenra' pears in regular air cold storage. The fruit were treated with 1-MCP inside sealed plastic boxes at $20{ }^{\circ} \mathrm{C}$, for 14 hours, and then transferred to cold storage $\left(0^{\circ} \mathrm{C} \pm\right.$ $1{ }^{\circ} \mathrm{C} ; 85 \% \pm 5 \%$ UR), where they were kept during 30,60, 90, and 120 days. The assessed parameters were flesh firmness, DA index, $\mathrm{pH}$ of juice, titratable acidity and soluble solids. It was observed that 1-MCP was efficient in maintaining fruit flesh firmness of the studied cultivars kept in cold storage. Also, the degradation of chlorophyll in fruits of 'Abate Fetel' and 'Packham's' pear was reduced with 1-MCP application.
\end{abstract}

KEYWORDS: Pyrus communis, ethylene, ripening, fruit quality.

\section{INTRODUÇÃO}

O armazenamento em câmara fria é a prática mais comumente utilizada para retardar o amadurecimento de peras e prolongar sua disponibilidade comercial (GAMRASNI et al. 2010). Porém, o armazenamento a frio induz a síntese de enzimas envolvidas na biossíntese de etileno (VILLALOBOSACUÑA et al. 2011) o qual é responsável pela aceleração do amadurecimento e redução de firmeza de polpa (MARTIN et al. 2015), reduzindo o potencial de conservação pós-colheita de peras. Uma das estratégias para manutenção da qualidade e aumento no tempo de armazenamento de frutas climatéricas como a pera é a utilização do 1-MCP.

O 1-MCP é um inibidor da ação do etileno que age pela fixação preferencial e irreversível ao receptor do etileno (BLANKENSHIP \& DOLE 2003), reduzindo os efeitos do etileno procedente de fontes internas e externas, aumentando o tempo de armazenamento dos frutos no período pós-colheita (LIMA et al. 2005). 
Esse produto é não tóxico em baixas concentrações, além disso é eficiente na manutenção da qualidade de frutas (NANTHACHAl et al. 2007). A capacidade inibitória da maturação de forma satisfatória depende de muitos fatores, tais como, cultivar, a maturação dos frutos na colheita, as condições de armazenamento e comercialização (CALVO \& SOZZI 2004, EKMAN et al. 2004). A aplicação de $200 \mu \mathrm{g} \mathrm{L}$ L $^{-1}$ de 1-MCP possibilitou a conservação pós-colheita de peras 'Spadona' em atmosfera controlada por seis meses, mantedo-se a qualidade de fruta dentro dos padrões comerciais por duas semanas após a retirada da câmara (GAMRASNI et al. 2010).

Em adição à inibição da maturação, outros importantes benefícios do 1-MCP para peras são a inibição da escaldadura superficial, escurecimento de polpa e hematomas causados por impactos (CALVO \& SOZZI 2004, EKMAN et al. 2004). A aplicação comercial de 1-MCP em algumas cultivares de peras ainda é limitada, pois as frutas tratadas não recuperam a capacidade de amadurecimento (CHEN \& SPOTTS 2005), a qual é necessária para obter as características organolépticas desejadas pelos consumidores (VILLALOBOS-ACUÑA \& MITCHAM 2008).

O objetivo deste trabalho foi avaliar a influência do 1-metilciclopropeno na qualidade pós-colheita de peras 'Abate Fetel', 'Packham's' e 'Tenra' armazenadas em câmara fria.

\section{MATERIAL E MÉTODOS}

O experimento foi realizado na safra 2011-2012 no Laboratório de Fruticultura de Clima Temperado na Faculdade de Agronomia Eliseu Maciel (FAEM), da Universidade Federal de Pelotas (UFPel), no município do Capão do Leão, RS. As frutas foram obtidas de pereiras das cultivares 'Abate Fetel', 'Packham's' e 'Tenra', com nove anos de idade, no Pomar Experimental da Palma, localizado no município do Capão do Leão (Latitude $31^{\circ} 52^{\prime}$ 00" S; Longitude 52 $21^{\circ}$ ' 24" W Greenwich; Altitude: 13,24 m.).

As frutas das cultivares 'Abate Fetel' e 'Tenra' foram colhidas dia 16 de janeiro de 2012 e as frutas da cultivar 'Packham's Triumph' dia 29 de janeiro de 2012. Na ocasião da colheita foi realizada a caracterização da maturação de cada cultivar (firmeza, índice DA e sólidos solúveis). Os valores de firmeza, índice DA e sólidos solúveis para as cultivares estudas, no momento da colheita, foram: 'Tenra' - 51,99N, Índice DA 1,98 e 10,28 `brix; 'Packham's Triumph' - 64,05 N, Índice DA 1,91 e 14,44 'brix; 'Abate Fetel' $68,71 \mathrm{~N}$, Índice DA 1,76 e 13,45 'brix. Foram utilizadas quatro repetições por tratamento, com cinco frutas cada. Os tratamentos utilizados foram: 1) controle - sem 1-MCP e; 2) $300 \mu \mathrm{g} \mathrm{L}^{-1}$ de 1-MCP. As frutas foram tratadas com 1-MCP no interior de caixas plásticas vedadas, à temperatura de $20{ }^{\circ} \mathrm{C}$, por 14 horas. As frutas do tratamento controle foram submetidas às mesmas condições, ou seja, ficaram no interior de caixas plásticas vedadas sem a aplicação de 1-MCP. Utilizou-se o 1-metilciclopropeno (1-MCP), nome comercial SmartFresh ${ }^{\circledR}$ (Rohm and Hass Inc.).

As frutas foram colocadas em câmara fria logo após terem sido retiradas das caixas vedadas. Os períodos de armazenamento foram: 'Tenra': 1) 30 dias de armazenamento em frio (AF; $0{ }^{\circ} \mathrm{C} \pm 1{ }^{\circ} \mathrm{C}$ e $85 \% \pm$ $5 \%$ de Umidade Relativa (UR)); 2) 30 dias $\mathrm{AF}+3$ dias em temperatura ambiente $\left(\mathrm{TA} ; 20{ }^{\circ} \mathrm{C} \pm 1{ }^{\circ} \mathrm{C}\right.$ e $70 \%$ UR); 3) 60 dias AF; 4) 60 dias AF + 3 dias TA; 5) 90 dias AF; 6) 90 dias AF + 3 dias TA; 7) 120 dias AF; 8) 120 dias AF + 3 dias TA; 'Abate Fetel' e 'Packham's': 1) 60 dias AF; 2) 60 dias AF + 3 dias TA; 3) 120 dias AF; 4) 120 dias AF + 3 dias TA.

As análises físico-químicas foram realizadas ao final de cada período de armazenagem. As variáveis analisadas foram: a) Índice DA, mensurado com espectrofotômetro portátil DA-Meter (Turoni, Itália), em dois pontos opostos do fruto; b) Sólidos solúveis (SS - ${ }^{\circ}$ Brix) - mensurado com refratômetro digital modelo PR-32 (Atago Co., Tokio, Japão), com compensação automática de temperatura; c) Firmeza de Polpa (FP) - expressa em Newtons (N), determinada com auxílio de penetrômetro digital modelo 53205 (TR Di Turoni, Forly, Itália) com ponteira de $8 \mathrm{~mm}$, em dois pontos opostos, na região equatorial das frutas, d) $\mathrm{pH}$ - determinado por $\mathrm{pH}$ metro digital de bancada microprocessado modelo DLA-pH; e) Acidez titulável (AT - \% ácido málico) - A acidez titulável (AT) foi mensurada por titulação do suco misturado à água destilada a uma razão de $1: 10$, com solução de $\mathrm{NaOH} 0,1 \mathrm{~N}$ a um ponto final de $\mathrm{pH} 8,1$ utilizando um $\mathrm{pH}$ metro digital microprocessado modelo DLA-pH. Para o cálculo da mesma, que é expressa em porcentagem de ácido málico, utilizou-se a seguinte fórmula:

$$
\% \text { ácido málico }=(\mathrm{Vg} \times \mathrm{N} \times \text { Meq. Ac. } \times 100) / \mathrm{A}
$$

onde: $\mathrm{Vg}=$ volume de $\mathrm{NaOH}$ gasto $(\mathrm{mL}) ; \mathrm{N}=$ normalidade da solução de $\mathrm{NaOH}$ utilizada $=0,1 \mathrm{~N}$; Meq. Ac. = miliequivalente ácido, que para o ácido málico é 0,06705; $\mathrm{A}=$ volume de suco utilizado $(10 \mathrm{~mL})$.

O delineamento experimental foi completamente casualizado, constituindo fatorial $4 \times 2$ (períodos de 
armazenamento $\mathrm{x}$ tratamentos) para as cultivares 'Abate Fetel' e' Packham's' e $8 \times 2$ para Tenra. As análises estatísticas foram realizadas utilizando o programa $R$ ( $R$ CORE TEAM 2014), com o pacote ExpDes (FERREIRA et al. 2013). A análise de variância foi realizada pelo teste $F$ e, quando este foi significativo, os dados foram submetidos à comparação de médias pelo teste de Tukey ao nível de $5 \%$ de probabilidade de erro.

\section{RESULTADOS}

Para a cultivar Abate Fetel, foi observada interação entre os fatores período de armazenamento e tratamento com 1-MCP para a variável firmeza de polpa (FP) (Tabela 1). Considerando-se o período de armazenamento no nível controle, a firmeza foi maior apenas aos 60 dias AF. Já para o nível 1-MCP, a firmeza foi menor apenas aos 120 dias AF + 3 dias TA (Tabela 1). Considerando-se o tratamento com 1MCP em cada período de armazenamento, os frutos submetidos a aplicação de 1-MCP apresentaram maior firmeza que o controle em todos os períodos de armazenamento, exceto aos 60 dias AF (Tabela 1). Interação significativa também foi observada para a variável índice DA. Para os frutos do controle, os menores valores foram observados aos 120 dias AF e 120 dias AF+ 3 dias TA, enquanto que no nível 1MCP, apenas aos 120 dias AF o índice DA foi menor que aos 60 dias AF.

Tabela 1. Firmeza, índice DA, pH do suco, acidez titulável e sólidos solúveis de peras 'Abate Fetel' e 'Packham's' tratadas em pós-colheita com com $300 \mu \mathrm{g} \mathrm{L}^{-1}$ de 1-metilciclopropeno (1-MCP), em diferentes períodos de armazenamento.

Table 1. Firmness, $D A$ index, juice $\mathrm{pH}$, titratable acidity and soluble solids of 'Abate Fetel' and 'Packham's' pears treated in post-harvest with $300 \mu \mathrm{g} \mathrm{L-1}$ of 1-methylcyclopropene (1-MCP), at different storage periods.

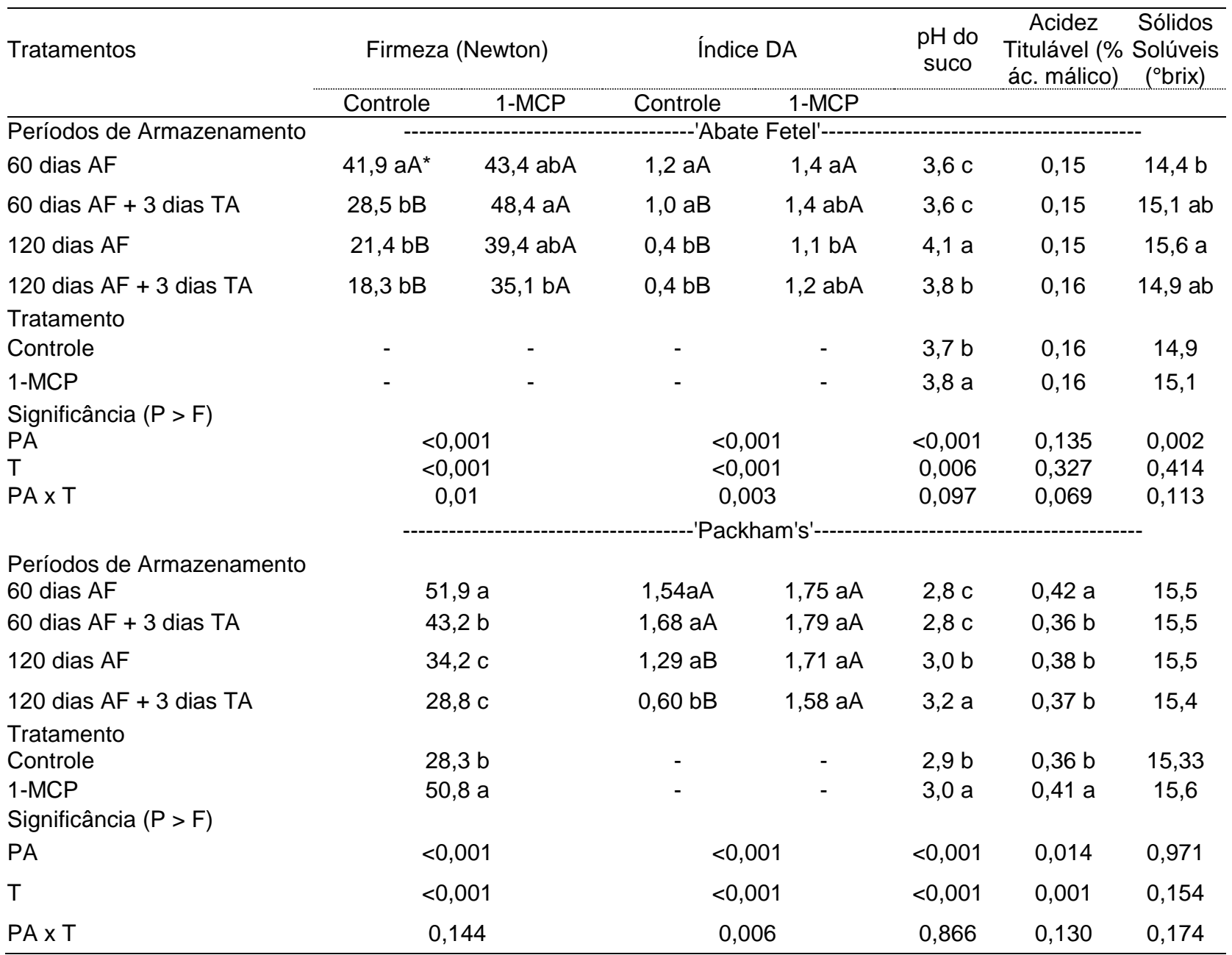

*Médias seguidas de letras distintas, minúsculas na coluna e maiúsculas na linha, diferem significativamente pelo teste de Tukey $(p<0,05)$. AF: armazenamento a frio; TA: temperatura ambiente.

À exceção do período 60 dias AF, frutos tratados com 1-MCP apresentaram maior índice DA do que o controle (Tabela 1). 
Considerando-se a cultivar 'Packham's', não foi observada interação significativa para a variável firmeza de polpa. Nos períodos de armazenamento, a firmeza foi maior aos 60 dias AF e menor com 120 dias AF e 120 dias AF +120 dias AF + 3 dias TA. Ainda, frutos tratados com 1-MCP apresentaram maior firmeza de polpa que o controle (Tabela 1). Já para a variável índice DA, foi observada interação significativa entre os fatores. Considerando-se o fator período de armazenamento no nível controle de tratamento, o índice DA aos 120 dias AF + 3 dias TA foi menor que os demais, sendo que dentro de 1-MCP, não foram observadas diferenças. Considerando-se cada período de armazenamento, frutos tratados com 1-MCP apresentaram maior índice DA do que o controle aos 120 dias AF e 120 dias AF + 3 dias TA (Tabela 1).

Para as variáveis pH do suco, acidez titulável e sólidos solúveis, não foi observada interação entre os fatores períodos de armazenamento e tratamento em ambas as cultivares (Tabela 1). Considerando-se 0 fator período de armazenamento, o pH do suco foi menor aos 60 dias AF e 60 dias AF + 3 dias TA, enquanto que o tratamento com 1-MCP resultou em maior $\mathrm{pH}$ do suco, tanto para 'Abate Fetel' quanto para 'Packham's' (Tabela 1). A acidez titulável diferenciou apenas para 'Packham's', em que o maior valor foi observado aos 60 dias AF em relação aos demais períodos de armazenamento e com 1-MCP em relação ao controle (Tabela 1). Os sólidos solúveis diferenciaram na cultivar 'Abate Fetel' entre os períodos de armazenamento, sendo que aos 120 dias AF o teor de sólidos solúveis foi maior que aos 60 dias AF (Tabela 1).

Para a cultivar 'Tenra', foi observada interação entre os fatores para as variáveis firmeza de polpa e índice DA (Tabela 2). Considerando-se os períodos de armazenamento no nível controle de tratamento, os menores valores de firmeza foram observados aos 90 dias $\mathrm{AF}+3$ dias TA, 120 dias AF e 120 dias AF +3 dias TA, enquanto que dentro do nível 1-MCP foram aos 90 dias AF +3 dias TA e 120 dias AF +3 dias TA. Observando-se o comportamento dos tratamentos em cada período de armazenamento, frutos tratados com 1-MCP apresentaram maior firmeza que o controle em todos os períodos exceto 30 dias AF, 60 dias AF e 90 dias AF (Tabela 2). Comportamento semelhante foi observado para o índice DA, em que o menor valor foi observado aos 120 dias $A F+3$ dias TA, para ambos o controle e 1-MCP. Frutos em que foi realizada aplicação de 1-MCP apresentaram maior índice DA aos 30 dias AF + 3 dias TA, 90 dias AF e 120 dias AF (Tabela 2). Para as demais variáveis nessa cultivar não foi observada interação significativa entre os fatores. $\mathrm{O}$ pH do suco foi menor aos 120 dias $\mathrm{AF}+3$ dias TA e nos frutos do controle. A acidez titulável não diferenciou entre os períodos de armazenamento, mas foi observada maior acidez titulável nos frutos do controle (Tabela 2).

\section{DISCUSSÃO}

A firmeza considerada ideal para consumo e comercialização situa-se entre 30-40N (MITCHAM \& MITCHELL 2007). Logo, observa-se que o tratamento com 1-MCP foi eficiente na manutenção da firmeza dentro dos padrões comerciais de peras 'Abate Fetel' e 'Tenra' por até 120 dias AF +3 dias TA, enquanto frutos não tratados mantiveram-se nesses padrões até 60 dias $A F+3$ dias $T A$, ou seja, redução no potencial de armazenamento de aproximadamente 50\%. Resultados semelhantes foram observados em peras 'Spadona' tratadas com $200 \mathrm{~g} \mathrm{~L} \mathrm{~L}^{-1}$, as quais apresentaram firmeza aceitável por até duas semanas em temperatura ambiente $\left(\sim 20^{\circ} \mathrm{C} ; 70 \%\right.$ UR) após seis meses de armazenamento em atmosfera controlada (GAMRASNI et al. 2010). Efeito semelhante foi observado por EKMAN et al. (2004) em peras 'Bartlett', os quais observaram maior redução da firmeza de polpa em frutos não tratados com 1-MCP.

O armazenamento em câmara fria é a prática mais comum para retardar o amadurecimento de peras e prolongar sua disponibilidade comercial (GAMRASNI et al. 2010), porém quando o mesmo é utilizado isoladamente não é suficiente para manter a qualidade dos frutos por longos períodos. Logo a combinação com técnicas como a aplicação de 1-MCP para reduzir a síntese de etileno pode aumentar o potencial de armazenamento de peras. Isso pode ser observado nos dados obtidos com as peras 'Abate Fetel' e 'Tenra', em que a firmeza após 60 dias AF ('Abate Fetel') e 30, 60 e 90 dias AF ('Tenra') não diferiu entre o controle e 1-MCP, mas aos 120 dias AF os frutos tratados com 1-MCP estavam mais firmes. Esse resultado mostra que a aplicação de 1-MCP potencializa o período de armazenamento a frio de peras 'Abate Fetel' e 'Tenra'. Resultados semelhantes foram observados por EKMAN et al. (2004), os quais observam que a firmeza de peras 'Bartlett' tratadas com $500 \mu \mathrm{g} \mathrm{L} \mathrm{L}^{-1} 1$-MCP logo após a retirada da câmara foi maior que o controle apenas após 170 dias de armazenamento. Porém, esses autores observaram que a manutenção da firmeza após 40 dias AF e 3 dias TA $\left(20^{\circ} \mathrm{C}\right)$ foi maior nos frutos tratados com $500 \mu \mathrm{g} \mathrm{L}{ }^{-1} 1$-MCP. Esse efeito também foi observado no presente estudo com as peras 'Tenra' e 'Abate Fetel'.

O amarelecimento da casca, o qual envolve degradação da clorofila, é parte de um complexo 
processo fisiológico envolvido no amadurecimento responsável pelas mudanças na cor, textura e sabor e aroma de frutos (ALEXANDER \& GRIERSON 2002). O amarelecimento da casca em excesso geralmente resulta na depreciação do preço de peras (CHENG et al. 2012). No presente trabalho, para todas as cultivares estudas foi possível observar que frutos tratados com 1-MCP apresentaram maior índice DA (o qual mensura de maneira não-destrutiva a degradação da clorofila) em relação ao controle e ao longo dos períodos de armazenamento. Na cultivar 'Packham's' esse efeito foi bastante pronunciado, pois não houve diferenças no índice DA entre os períodos de armazenamentos, ou seja, a coloração verde das peras aos 60 dias $\mathrm{AF}$ foi similar àquelas armazenadas por 120 dias $\mathrm{AF}+3$ dias TA.

Tabela 2. Firmeza, índice DA, pH do suco, acidez titulável e sólidos solúveis de peras 'Tenra' tratadas em pós-colheita com $300 \mu \mathrm{g} \mathrm{L}^{-1}$ de 1-metilciclopropeno (1-MCP), em diferentes períodos de armazenamento.

Table 2. Firmness, DA index, juice $\mathrm{pH}$, titratable acidity and soluble solids of 'Tenra' pears treated in postharvest with $300 \mu \mathrm{g} \mathrm{L}^{-1}$ of 1-methylcyclopropene (1-MCP), at different storage periods.

\begin{tabular}{|c|c|c|c|c|c|c|c|}
\hline \multirow[t]{2}{*}{ Tratamentos } & \multicolumn{2}{|c|}{ Firmeza (Newton) } & \multicolumn{2}{|c|}{ Índice DA } & \multirow{2}{*}{$\begin{array}{l}\mathrm{pH} \text { do } \\
\text { suco }\end{array}$} & \multirow{2}{*}{$\begin{array}{c}\text { Acidez } \\
\text { Titulável (\% } \\
\text { ác. málico) }\end{array}$} & \multirow{2}{*}{$\begin{array}{c}\text { Sólidos } \\
\text { Solúveis } \\
\text { ('brix) }\end{array}$} \\
\hline & Controle & 1-MCP & Controle & 1-MCP & & & \\
\hline \multicolumn{8}{|c|}{ Períodos de Armazenamento } \\
\hline 30 dias $\mathrm{AF}$ & $48,1 \mathrm{aA}^{*}$ & $46,2 \mathrm{abA}$ & $1,8 \mathrm{aA}$ & $1,8 \mathrm{aA}$ & $3,4 \mathrm{~b}$ & 0,36 & 11,8 \\
\hline 30 dias $\mathrm{AF}+3$ dias TA & $34,7 \mathrm{bB}$ & $50,9 \mathrm{aA}$ & $1,6 \mathrm{abB}$ & $1,8 \mathrm{aA}$ & $2,9 d$ & 0,35 & 11,7 \\
\hline $60 \vee A F$ & $43,2 \mathrm{aA}$ & $41,5 \mathrm{bcA}$ & $1,7 \mathrm{abA}$ & $1,7 \mathrm{abA}$ & $3,0 \mathrm{~d}$ & 0,34 & 11,5 \\
\hline 60 dias $\mathrm{AF}+3$ dias TA & 29,3 bcB & $47,3 \mathrm{abA}$ & $1,5 \mathrm{bcA}$ & $1,4 \mathrm{bcA}$ & $2,9 \mathrm{~d}$ & 0,30 & 11,7 \\
\hline 90 dias $\mathrm{AF}$ & $34,6 \mathrm{bA}$ & $36,9 \mathrm{~cd} A$ & $1,4 \mathrm{bcB}$ & $1,6 \mathrm{abcA}$ & $2,9 \mathrm{~d}$ & 0,35 & 11,5 \\
\hline 90 dias $\mathrm{AF}+3$ dias TA & $22,2 \mathrm{cB}$ & $32,9 \mathrm{dA}$ & $1,2 \mathrm{~cd} A$ & $1,4 \mathrm{cA}$ & $3,0 \mathrm{~d}$ & 0,33 & 11,5 \\
\hline 120 dias AF & $25,5 \mathrm{cB}$ & $34,6 \mathrm{~cd} A$ & $0,9 \mathrm{deB}$ & $1,4 \mathrm{bcA}$ & $3,6 \mathrm{a}$ & 0,34 & 11,6 \\
\hline $\begin{array}{l}120 \text { dias } \mathrm{AF}+3 \text { dias } \mathrm{TA} \\
\text { Tratamento }(\mathrm{T})\end{array}$ & $24,6 \mathrm{cB}$ & $30,3 \mathrm{dA}$ & 1,1 eA & $1,1 \mathrm{dA}$ & $3,2 \mathrm{c}$ & 0,31 & 11,6 \\
\hline Controle & - & - & - & - & $3,1 \mathrm{a}$ & $0,36 \mathrm{a}$ & 11,7 \\
\hline $1-\mathrm{MCP}$ & - & - & - & - & $3,2 \mathrm{~b}$ & $0,32 \mathrm{~b}$ & 11,6 \\
\hline \multicolumn{8}{|l|}{ Significância $(P>F)$} \\
\hline PA & \multicolumn{2}{|c|}{$<0,001$} & \multicolumn{2}{|c|}{$<0,001$} & $<0,001$ & 0,303 & 0,962 \\
\hline $\mathrm{T}$ & \multirow{2}{*}{\multicolumn{2}{|c|}{$\begin{array}{l}<0,001 \\
<0,001\end{array}$}} & \multicolumn{2}{|c|}{$<0,001$} & $<0,001$ & 0,006 & 0,389 \\
\hline PA $\times T$ & & & \multicolumn{2}{|c|}{0,005} & 0,087 & 0,794 & 0,836 \\
\hline
\end{tabular}

${ }^{*}$ Médias seguidas de letras distintas, minúsculas na coluna e maiúsculas na linha, diferem significativamente pelo teste de Tukey $(p<0,05)$. AF: armazenamento a frio; TA: temperatura ambiente.

Esse resultado é extremamente importante, pois a manutenção da coloração, associada a redução na perda de firmeza pode resultar em maior preço de venda ao produtor, em um período de baixa oferta da fruta. CHENG et al. (2012) em estudo sobre a degradação de clorofila da casca de peras chinesas 'Emerald', observaram que a aplicação de $1000 \mu \mathrm{g} \mathrm{L}^{-1}$ de 1-MCP, reduziu a degradação de clorofila da casca, mantendo a coloração verde dos frutos por um período de tempo mais longo.

Foi observada redução na acidez titulável na cultivar 'Packham's' ao longo do período de armazenamento. Frutos de 'Packham's' e 'Tenra' tratados com 1-MCP apresentaram maior acidez titulável do que os não tratados. Essa condição provavelmente seja efeito dos níveis mais altos de etileno nos frutos que não receberam tratamento com 1-MCP, proporcionando maior degradação dos ácidos orgânicos pelo processo respiratório (BRACKMANN et al. 2009).

\section{CONCLUSÃO}

O 1-MCP, na dose de $300 \mu \mathrm{g} \mathrm{L}-1$, é eficiente na manutenção da firmeza de polpa de peras 'Abate Fetel', Packham's' e 'Tenra', armazenadas em câmara fria.

A degradação de clorofila de peras 'Abate Fetel' e 'Packham's', armazenadas em câmara fria, é reduzida com a utilização do 1-MCP, na dose de $300 \mu \mathrm{g} \mathrm{L}^{-1}$.

\section{REFERÊNCIAS}

ALEXANDER L \& GRIERSON D. 2002. Ethylene biosynthesis and action in tomato: A model for climacteric fruit ripening. Journal of Experimental Botany 53: 2039-2055.

BLANKENSHIP SM \& DOLE JM. 2003. 1-Methylcyclopropene: a review. Postharvest Biology and Technology 28: 1-25.

BRACKMANN A et al. 2009. Absorção de 1-metilciclopropeno aplicado em maçãs 'Royal Gala' armazenadas em atmosfera refrigerada juntamente com madeira. Ciência Rural 39: 1676-1681. 
CALVO G \& SOZZI GO. 2004. Improvement of postharvest storage quality of 'Red Clapp's' pears by treatment with 1methylcyclopropene at low temperature. The Journal of Horticultural Science and Biotechnology 79: 930-934.

CHEN PM \& SPOTTS R. 2005. Changes in ripening behaviors of 1-MCP treated d'Anjou pears after storage. International Journal of Fruit Science 5: 3-18.

CHENG $\mathrm{Y}$ et al. 2012. Effects of 1-MCP on chlorophyll degradation pathway-associated genes expression and chloroplast ultrastructure during the peel yellowing of Chinese pear fruits in storage. Food Chemistry 135: 415-422.

EKMAN JH et al. 2004. Interactions between 1-MCP concentration, treatment interval and storage time for 'Bartlett' pears. Postharvest Biology and Technology 31: 127-136.

FERREIRA EB et al. 2013. ExpDes: Experimental Designs Package. R package version 1.1.2.

GAMRASNI D et al. 2010. 1-Methylcyclopropene (1-MCP) application to Spadona pears at different stages of ripening to maximize fruit quality after storage. Postharvest Biology and Technology 58: 104-112.

LIMA LC et al. 2005. Controle do amadurecimento de banana 'Prata-Anã' armazenada sob refrigeração e atmosfera modificada passiva com o uso do 1-Metilciclopropeno. Ciência e Agrotecnologia 29: 476-480.

MARTIN MS et al. 2015. Indução do amadurecimento de peras 'Rocha' submetidas à baixa temperatura e à aplicação de etileno. Pesquisa Agropecuária Brasileira 50: 273-281.

MITCHAM EJ \& MITCHELL G. 2007. Conditioning and ripening of Bartlett pears. In: MITCHAM EJ \& ELKINS RB. (Eds). Pear: production and handling manual. California: University of California. p. 179-181.

NANTHACHAI NN et al. 2007. Absorption of 1-MCP by fresh produce. Postharvest Biology and Technology 43: $291-297$.

R CORE TEAM. 2014. R: A language and environment for statistical computing. Vienna, Austria: R Foundation for Statistical Computing. Available at: https://www.r-project.org/. Access on: Jul. 17, 2015.

VILLALOBOS-ACUÑA M \& MITCHAM EJ. 2008. Ripening of European pears: the chilling dilemma. Postharvest Biology and Technology 49: 187-200.

VILLALOBOS-ACUÑA MG et al. 2011. Effect of maturity and cold storage on ethylene biosynthesis and ripening in 'Bartlett' pears treated after harvest with 1-MCP. Postharvest Biology and Technology 59: 1-9. 